\title{
Congestion Driven Incremental Placement Algorithm for Standard Cell Layout
}

\author{
Zhuoyuan Li, Weimin Wu, Xianlong Hong \\ Department of Computer Science and Technology \\ Tsinghua University, Beijing, 100084, P.R.China
}

\begin{abstract}
Congestion minimization is the least understood in placement objectives, however, it models routability most accurately. In this paper, a new incremental placement algorithm C-ECOP for standard cell layout is presented to reduce routing congestion. Congestion estimation is based on a new routing model and a more accurate cost function. An integer linear programming (ILP) problem is formulated to determine cell flow direction and avoid the conflictions between adjacent congestion areas. Experimental results show that the algorithm can considerably reduce routing congestion and preserve the performance of the initial placement with high speed.
\end{abstract}

Key words: congestion, standard cell, incremental placement

\section{Introduction}

As VLSI technology advances, the system complexity continues to increase and physical design is getting more and more difficult. With the advent of overcell routing, the goal of every place and route methodology has been to utilize all available active area to prevent spilling of routes into channels. It is the overflow of routes that account for an increase in area. Further heuristic method should be applied in placement to manage local congestion to enhance and improve the latter route ability.

Traditional placement objectives involve reducing net-cut costs or minimizing wire length [1-2]. Because of its constructive nature, min-cut based strategies minimize the number of net crossings but fail to distribute them uniformly [3]. For the same reason, traditional placement schemes which are based mainly on wirelength minimization can not adequately account for congestion. Reducing net-cut and minimizing wirelength only help reduce the routing demand globally but do not prevent causing local routing congestion. How to estimate and reduce congestion in placement is not well studied. Congestion-driven placement based on multi-partitioning was proposed in [4]. It uses the actual congestion cost calculated from precomputed Steiner trees to minimize the congestion of the chip. However, the number of partitions is limited due to the excessive computational load.

Wang et al. [8-10] proposed a consistent routing model defined by demand/supply relationship. Experimental results show that the congestion objective is very ill behaved. So it adapts a post processing approach

\footnotetext{
* The work was supported by the National Natural Science Foundation of China (NSFC) 60121120706, the National Natural Science Foundation of USA (NSF) CCR-0096383, Hi-Tech Research \& Development (863) Program of China 2002AA1Z1460 and The National Foundation Research (973) Program of China G1998030403
}

after placement to reduce congestion. But the demand/supply congestion model and bounding-box routing estimation is too simple and will affect the final result. Since congestion and wirelength are globally consistent, Jason et al. [11-12] considered improving local congestion with incremental placement. However, how to minimize the wirelength changes caused by congestion reducing and maintain the metrics of the initial placement is very difficult.

In this paper, an incremental placement algorithm C-ECOP for improving local congestion is proposed. It first estimates the routing congestion through a new route model. Then it constructs an integer linear programming (ILP) to move cells to reduce congestion. Finally it adjusts the positions of cells to resolve overlap. The rest of this paper is organized as follows. Section 2 describes the routing estimation and congestion measurement used in this work. The algorithm C-ECOP is presented in section 3 . Section 4 gives the experimental results to show the effectives of our algorithm. Section 5 is the conclusion.

\section{Congestion Estimation in Placement}

\subsection{Congestion Cost}

The congestion cost is defined based on the global bin concept. We partition a given chip into several rectangular regions, each of which is called a global bin. The boundaries of global bins are called global bin edges as shown in Fig.1. The congestion is "related" to the number of crossings between routed nets and global bin edges.

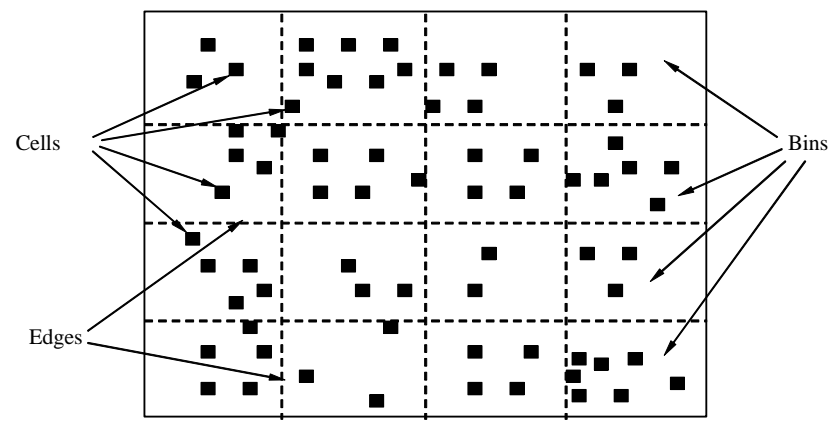

Fig.1 Bin Structure

Given a detailed placement, all the cells and pads have fixed positions on the chip. We can use a "router" to route all the nets. The router can be a very simple global router or even a bounding box router. Therefore, for each global edge $e$, the routing demand of $e, d_{e}$, can be defined as the number of the nets crossing $e$. The routing supply of $e, s_{e}$, is known easily from the technology parameter. A 
global edge is congested if and only if routing demand $d_{e}$ exceeds the routing supply $s_{e}$. The overflow is defined as follows:

$$
\text { overflow }(e)=\left\{\begin{array}{cc}
0 & \left(d_{e} \leq s_{e}\right) \\
d_{e}-s_{e} & \left(d_{e}>s_{e}\right)
\end{array}\right.
$$

Then estimating the congestion of a global bin can be replaced by computing the total overflow of the global edges as (2) and cost function is defined as (3). Most of the algorithms for reducing congestion [6-10] estimate congestion like this.

$$
\begin{aligned}
& \operatorname{con}\left(b_{i j}\right)=\sum_{k=1}^{4} \text { overflow }\left(e_{k}\right) \\
& \cos t_{c}=\sum_{i=1}^{n} \sum_{j=1}^{n} \operatorname{con}\left(b_{i j}\right)
\end{aligned}
$$

This method to estimate congestion is ill behaved. It can only detect the congestion area that is crossed by a global edge. Actually, the congestion area can be in any place on the chip and it may just locate inside a global bin. Table 1 gives the result of the congestion estimation on a circuit CNT100 with the method above. The chip is partitioned into $4 \times 4,5 \times 5,6 \times 6$ and $7 \times 7$ global bins. It is shown that the total number of congest areas (TNC.), the total demand (TD.) changed due to different partition. The max demand (MD.) even increases when the partition amount increases from $4 \times 4$ to $5 \times 5$. Because the global edges become shorter, it seems that the routing demand crossing the edge should decrease.

Table 1. Estimation of Congestion on CNT100

\begin{tabular}{|l|l|l|l|l|l|}
\hline Grids & $\begin{array}{l}\text { Supply } \\
(\mathrm{H} / \mathrm{V})\end{array}$ & $\begin{array}{l}\text { TNC. } \\
(\mathrm{H} / \mathrm{V})\end{array}$ & $\begin{array}{c}\text { TD. } \\
(\mathrm{H} / \mathrm{V})\end{array}$ & $\begin{array}{l}\text { MD. } \\
(\mathrm{H} / \mathrm{V})\end{array}$ & $\begin{array}{l}\text { MO.** } \\
(\mathrm{H} / \mathrm{V})\end{array}$ \\
\hline $4 \times 4$ & $19 / 12$ & $0 / 1$ & $261 / 198$ & $\mathbf{1 8} / \mathbf{1 3}$ & $0 / 1$ \\
\hline $5 \times 5$ & $15 / 10$ & $4 / 4$ & $269 / 192$ & $\mathbf{2 0 / 1 4}$ & $5 / 4$ \\
\hline $6 \times 6$ & $13 / 8$ & $2 / 8$ & $322 / 226$ & $\mathbf{1 7 / 1 1}$ & $4 / 3$ \\
\hline $7 \times 7$ & $11 / 7$ & $4 / 6$ & $391 / 251$ & $\mathbf{1 8} / \mathbf{8}$ & $7 / 1$ \\
\hline
\end{tabular}

*H: Horizontal V: Vertical **MO.: Max Overflow

From the table we can see that the total demand increases when partition amount increases. It is because that the nets inside a larger global bin may become cross a global edge when the chip be partitioned with smaller bins. So the vertical and horizontal congestion estimation of a global bin in our algorithm is defined as follows:

$$
\begin{gathered}
\text { con }_{v}\left(b_{i j}\right)=\omega_{1} r_{v}\left(b_{i j}\right)+\omega_{2} \sum_{k=1}^{2} \text { overlfow }_{v}\left(e_{k}\right) \\
\text { con }_{h}\left(b_{i j}\right)=\omega_{1} r_{h}\left(b_{i j}\right)+\omega_{2} \sum_{k=1}^{2} \text { overlfow }_{h}\left(e_{k}\right)
\end{gathered}
$$

where $r_{v}$ and $r_{h}$ are the vertical and horizontal routing demand inside $b_{i j}$. It can be obtained by the routing model described in 2.2. $\omega_{1}$ and $\omega_{2}$ are the weights.

\subsection{Routing Estimation Model}

When we are performing congestion reducing, we need to estimate congestion of placement incrementally. A global router is needed here. Obviously, the more accurate is the router, the more accurate is the estimation at the placement stage. Routing with a real global router will provide an accurate congestion estimation. But it will be very time consuming and could not be applied in incremental placement algorithm. Routing with a simple routing model such as the bounding box model will be very fast. But the bounding box model may be far different with the characteristics of the detailed router so that it causes a bad estimation. Wang et al. verify the bounding box model in [8] and prove that it does not correlate with the real router and could not be applied. So it is critical that the algorithm for this application is accurate while maintaining computational efficiency.

A new star model proposed in [6] is used here. It first computes and adjusts the coordinate of the net center. The vertical and horizontal possible route paths connecting each cell to the center are on the edges of a rectangle whose two vertexes locate on the cell and the center. Route possibility on each path is 0.5 . Then all the route possibility on the same path is added up and could not exceed 1 as shown in Fig. 2. Experimental results show that the new star model is very close to the real routing in practice [6].
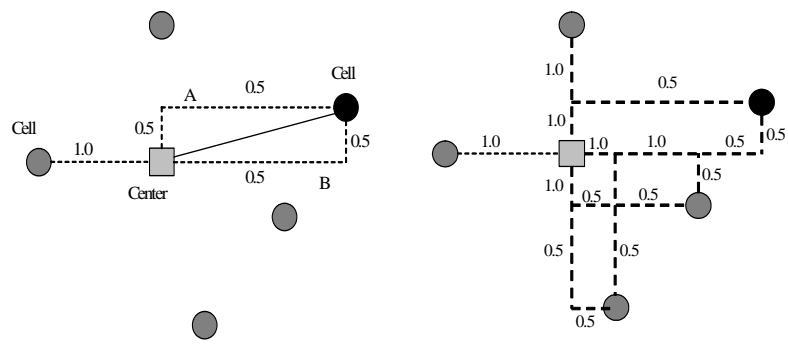

Fig.2 Routing Estimation with new star model

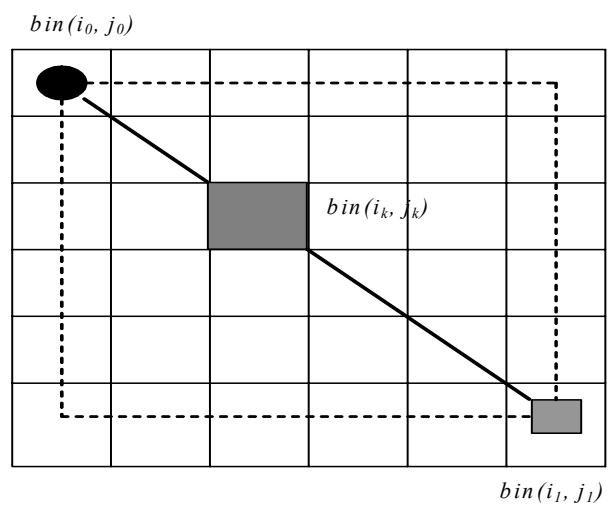

Fig.3 Route Probability

This model has an obvious drawback. The possible route path between a cell and the net center does not necessarily locate on the rectangle edges. It may cross any 
bin edges inside the rectangle as shown in Fig.3.

If cell $C_{k}$ is in $\operatorname{bin}\left(i_{0}, j_{0}\right)$, the net center is in $\operatorname{bin}\left(i_{1}, j_{l}\right)$, all the possible routes crossing the left edge of $\operatorname{bin}\left(i_{k}, j_{k}\right)$ are those from $\operatorname{bin}\left(i_{0}, j_{0}\right)$ to $\operatorname{bin}\left(i_{k}-1, j_{k}\right)$, crossing the edge between $\operatorname{bin}\left(i_{k^{-}}-1, j_{k}\right)$ and $\operatorname{bin}\left(i_{k}, j_{k}\right)$, then from $\operatorname{bin}\left(i_{k}, j_{k}\right)$ to $\operatorname{bin}\left(i_{1}, j_{l}\right)$. It is in the symmetric form on the right edge of $\operatorname{bin}\left(i_{k}, j_{k}\right)$. So the route possibility crossing the left and right edges of $\operatorname{bin}\left(i_{k}, j_{k}\right)$ could be denoted as:

$$
\begin{gathered}
p_{l}(k)= \begin{cases}w_{k l} & i_{0} \leq i_{k} \leq i_{1} \\
0 & j_{0} \leq j_{k} \leq j_{1} \\
0 & \text { otherwise }\end{cases} \\
p_{r}(k)= \begin{cases}w_{k r} & i_{0} \leq i_{k} \leq i_{1} \\
0 & j_{0} \leq j_{k} \leq j_{1}\end{cases} \\
w_{k l}=C_{i_{k}-i_{0}-1+j_{k}-j_{0}}^{i_{k}-i_{0}-1} \times C_{i_{1}-i_{k}+j_{1}-j_{k}}^{i_{1}-i_{k}} / C_{i_{1}-i_{0}+j_{1}-j_{0}}^{i_{1}-i_{0}} \\
w_{k r}=C_{i_{k}-i_{0}+j_{k}-j_{0}}^{i_{k}-i_{0}} \times C_{i_{1}-i_{k}-1+j_{1}-j_{k}}^{i_{1}-i_{k}-1} / C_{i_{1}-i_{0}+j_{1}-j_{0}}^{i_{1}-i_{0}}
\end{gathered}
$$

And the possibility of crossing the top and bottom edges, which are denoted as $p_{t}(k)$ and $p_{b}(k)$, are computed in the symmetric form. The vertical and horizontal routing demand inside a global bin can be easily known from this approach. The running time of congestion estimation on some circuits through this approach is listed in Table 2. It is shown that this approach is so fast that it could be used in our algorithm.

Table 2. Running Time on Routing Estimation

\begin{tabular}{|l|l|l|c|c|}
\hline Circuits & \#cells & \#nets & Grids & Running time(s) \\
\hline Ibm01 & 12,036 & 11,507 & $30 \times 30$ & 0.57 \\
\hline Ibm02 & 19,062 & 18,429 & $40 \times 40$ & 1.95 \\
\hline Ibm03 & 21,924 & 21,621 & $50 \times 50$ & 2.05 \\
\hline Ibm04 & 26,346 & 26,163 & $50 \times 50$ & 1.93 \\
\hline Ibm05 & 28,146 & 28,446 & $60 \times 60$ & 4.48 \\
\hline
\end{tabular}

\section{Congestion Reducing through ILP}

\subsection{Overview}

Generally, minimizing congestion and minimizing wirelength conflict each other in local regions. The reducing of congestion means to sacrifice the wirelength. Incremental placement algorithm should achieve trade-off between congestion reducing and preserving the metrics, e.g. wirelength, of the initial placement.

The design flow is shown in Fig.4. The flow tendency of each cell is computed based on force driven by nets. Then cells could move due to the tendency to reduce congestion. An integer linear programming is formulated to deal with the conflicts between multiple congested regions. After that a post process is carried out to place the moved cells and resolve overlap. The iteration of the ECO flow stops when the congestion result is acceptable.

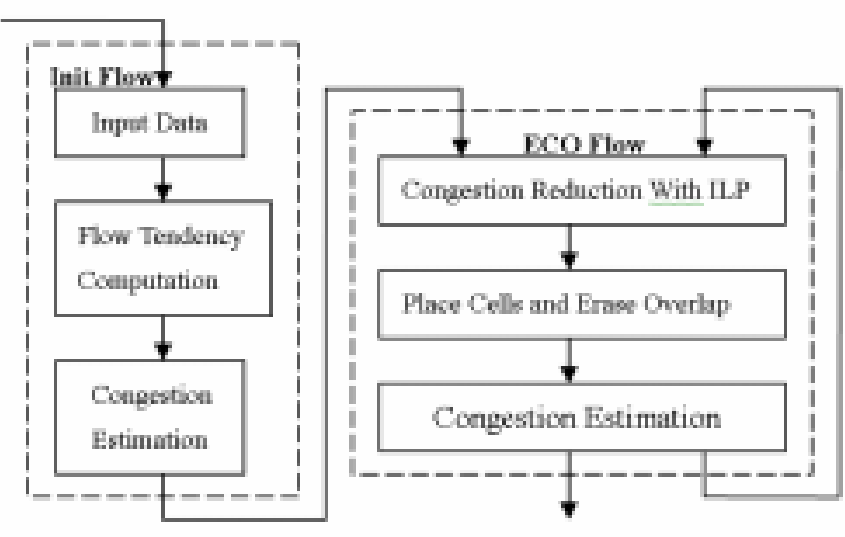

Fig.4 C-ECOP Algorithm

\subsection{Cell Flow Tendency Computation}

Based on routing estimation, we can identify the congestion bins on the chip. Each global bin whose congestion cost defined by (4) is greater than a certain threshold value or at least one of its global edge is overflowed is considered to be a congest bin. Cells in congest bins should move outside to decrease routing demand and achieve more routing resource. Which cells can be moved out so that the perturbation to the initial placement could be minimized is the key problem. We compute the flow tendency of each cell to deal with this.

The horizontal flow tendency of a cell $C_{k}$ in $\operatorname{bin}(i, j)$ is computed as follows:

$$
\begin{aligned}
& \text { xflow }_{l}\left(c_{k}\right)=\sum_{\text {net }_{k 1}} p_{l}(k)-\sum_{\text {net }_{k 2}} p_{r}(k)-\sum_{\text {net }_{k 3}} 1 \\
& \text { fflow }_{r}\left(c_{k}\right)=\sum_{\text {net }_{k 2}} p_{r}(k)-\sum_{\text {net }_{k 1}} p_{l}(k)-\sum_{\text {net }_{k 3}} 1
\end{aligned}
$$

where $n e t_{k l}$ are the nets whose center are in bins on the left, $n e t_{k 2}$ are the nets whose center are in bins on the right, $n e t_{k 3}$ are the nets whose center are in bin $b(i, j) . p_{l}(k)$ and $p_{r}(k)$ are defined in (5).

Xflow $_{l}$ denotes the gain of crossing nets decreased on the left edge of $\operatorname{bin}(i, j)$ when moving $C_{k}$ from $\operatorname{bin}(i, j)$ to $\operatorname{bin}(i-1, j)$. If it is positive, moving $C_{k}$ to the left will lead crossing nets on the edge decreased. It means a decrease in overflow on the edge. Xflow ${ }_{r}$ denotes the gain when moving right. And it is in the symmetric form for the vertical flow tendency. These parameters decide the move tendency of cells.

Moreover, the flow tendency of cells could be regarded as the net-cuts crossing the global edges. Generally speaking, reducing in net-cuts is consistent with reducing in wirelength, so moving cells according to the flow tendency will lead a decrease in wirelength. 


\subsection{Congestion Reducing Based on Integer Programming}

For a congest bin, cells inside should move out to reduce the nets inside and achieve more free space for routing. If $\operatorname{bin}(i, j)$ is vertical-congest, cells in it should move along the horizontal direction as shown in Fig.5:

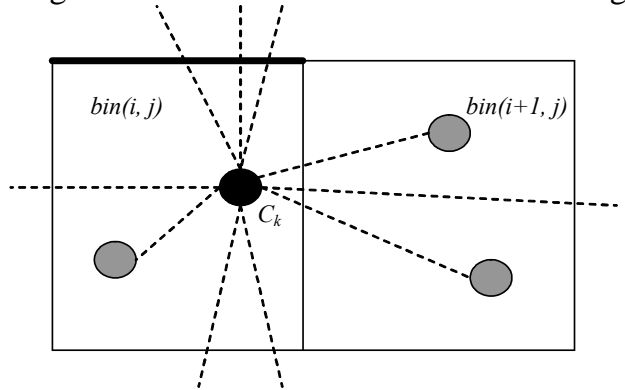

Fig.5 Move Cell to Reduce Congestion

When $C_{k}$ is moved into $\operatorname{bin}(i+1, j)$, the vertical route in $\operatorname{bin}(i, j)$ will decrease by:

$$
\operatorname{gain}_{v}\left(c_{k}\right)=\sum_{n e t_{k t}} p_{t}(k)+\sum_{n e t_{k b}} p_{b}(k)
$$

where $n t_{k t}$ are nets connected to $C_{k}$ and their centers are in the top bins. net $_{k b}$ are nets whose centers are in the bottom bins. $p_{t}(k)$ and $p_{b}(k)$ are the possibility of crossing the top and bottom edges of $\operatorname{bin}(i, j)$ estimated by the route model. Whether $C_{k}$ should move to the left or the right bin is according to $X$ flow $_{l}\left(C_{k}\right)$ and $X$ flow $_{r}\left(C_{k}\right)$. It assures that the horizontal congestion will not increase when reducing vertical congestion. To reduce the horizontal congest is in the symmetric method.

Note that the vertical route demand in $\operatorname{bin}(i+1, j)$ will increase after $C_{k}$ moves into it. This may cause unexpected congest regions. Furthermore, if $\operatorname{bin}(i+1, j)$ is already vertical-congested, moving $C_{k}$ will lead a more severe congest in it. An arbitrative mechanism is needed to deal with the conflicts between the congestion reducing among multiple congest regions. An integer programming problem is constructed to resolve it.

As mentioned above, only the cells with one or more positive flow tendency could be moved. Experimental results show that the conditions are too strict. For some circuits there are few cells could move to reduce congestion. So we relax the conditions here. For a congest bin $\operatorname{bin}(i, j)$, horizontal-movable cells should satisfy the following conditions:

$$
\text { xflow }_{l}\left(c_{k}\right)>t_{h} \text { or } \text { xflow }_{r}\left(c_{k}\right)>t_{h}
$$

where $t_{h}$ is a negative threshold. It means that we allow a little horizontal congest increase when reducing vertical congest. And the condition for vertical-movable cells is as follows:

$$
\text { xflow }_{t}\left(c_{k}\right)>t_{v} \text { or } \text { xflow }_{b}\left(c_{k}\right)>t_{v}
$$

It is obvious that only one equation in (8) and (9) could be positive. If $t_{h}$ equal 0 , only one of the two inequations in (11) may be satisfied, so for a movable cell $C_{k}$, its flow orientation is certain. When we relax the conditions, both of the two inequations in (11) may be satisfied. Then we randomly prescribe the moving orientation of $C_{k}$. Later we can see that this will assure high efficiency when solving the ILP problem.

The integer linear programming problem is defined as follows:

$$
\begin{array}{ll}
\text { s.t. } & \begin{array}{l}
\text { minimize } \\
\operatorname{con}_{h}\left(b_{i j}\right)-\sum_{C_{k}} m_{k i j} x_{k} \text { gain }_{h}\left(C_{k}\right) \leq C_{\max } \\
\operatorname{con}_{v}\left(b_{i j}\right)-\sum_{C_{k}} m_{k i j} y_{k} \text { gain }_{v}\left(C_{k}\right) \leq C_{\max }
\end{array} \\
& i, j=1,2, \ldots, N \\
& x_{k}+y_{k} \leq 1 \\
& x_{k}, y_{k} \in\{0,1\} \quad k=1,2, \ldots, M \\
& m_{k i j} \in\{-1,0,1\}
\end{array}
$$

where $C_{\max }$ is the maximum congestion degree over all the global bin.

$x_{k}$ could equal 1 only when (11) is satisfied.

$y_{k}$ could equal 1 only when (12) is satisfied.

$x_{k}+y_{k} \leq 1$ means that $C_{k}$ could not move vertically and horizontally at the same time.

$m_{k i j}$ denote which $C_{k}$ should be included in the inequations. For a movable $C_{k}$ in $\operatorname{bin}\left(i_{k}, j_{k}\right)$, because its vertical and horizontal flow orientation is certain, the bin it could move into is only. So the parameter could be defined as follows:

$$
m_{k i j}=\left\{\begin{array}{cr}
1 & i=i_{k} \text { and } j=j_{k} \\
-1 \text { bin }(i, j) & \text { is the bin } C_{k} \text { movesto } \\
0 & \text { otherwise }
\end{array}\right.
$$

$m_{k i j}$ equals 1 means route demand in $\operatorname{bin}\left(i_{k}, j_{k}\right)$ will reduce after moving $C_{k}$ out. It equals -1 means route demand in the destination bin will increase for the same amount. And the perturbation to the route probability in other bins is ignored. Experimental results show that it slightly affects the final results but considerably saves the running time.

Then for some bin, the number of its related cells is limited. Actually, it is only the movable cells inside the bin and in the four adjacent bins that may be included in the inequations. The ILP problem can be optimally solved. The problem solution determines the total number and destination bins of moved cells. A post processing is then carried out to place the cells and resolve overlap inside each bin.

\subsection{Post Processing}

After the congestion reducing process, some cells are redistributed among different global bins. These cells should be placed without overlap. An efficient algorithm is needed to adjust the positions of cells with the minimal perturbation to the initial placement. We use the W-ECOP algorithm [5] here to accomplish the process. For a cell 
need to be placed, it is inserted into the adjacent row and an optimal scheme to rearrange the cells in the row is found. If free space in the row can not accept the cell, a shifting path searching process is carried on to assure cells restrict in their neighboring area so that the performance of the circuit will be preserved.

\section{Experimental Results}

The algorithm has been implemented in C. All the experiments were done on a Sun E450 workstation with 4GB memory. To show the effectiveness and utility of our algorithm, a part of the experimental circuits are chosen from IBM-PLACE benchmark [13] placed with a wirelength-driven placer, Dragon [14]. Other set of circuits are from industry (Ultima Company). We compare the global routing results (overflow and wirelength) for the design before and after incremental placement.

Table 3 shows the results on the circuits from industry. As one can see, the congestion reducing approach considerably reduces the total overflow. It has a 50 percent cut down in average. And the total wirelength increased less than 0.1 percent compared to the initial placement. Some circuits even have a decrease in wrelength after placement. This indicates that the wirelength is not sacrificed much due to the reducing of congestion. It is owed to the wirelength optimization approach in W-ECOP algorithm.
Table 4 shows the results on the IBM-PLACE benchmarks placed with Dragon. Dragon has done wirelength and routability optimization by combining powerful hypergraph partitioning package with simulated annealing technique [14]. From the results we can see that the total overflow can be reduced continually through our method. And the optimization in wirelength is preserved. The algorithm is much faster on the benchmarks than on the industry circuits. The short amount of running time shows that our method can scale well for large circuits.

\section{Conclusion}

A new incremental placement algorithm for congestion alleviation is presented in this paper. The proposed algorithm automatically evaluates the routing congestion of a detailed placement with a fast and accurate routing estimation model. Congestion areas on the chip are relieved through cell moving. An integer linear programming (ILP) problem is formulated to resolve conflicts among multiple congest areas and avoid causing unexpected congest areas. After that an efficient algorithm for resolving overlap is used to ensure perturbing the initial placement the least. Experimental results demonstrate the effectiveness of the new approach.

Table 3 Experimental results on industry circuits

\begin{tabular}{|c|c|c|c|c|c|c|c|c|c|c|}
\hline \multirow{2}{*}{ Circuits } & \multirow{2}{*}{ \#Cells } & \multirow{2}{*}{ Grids } & \multirow{2}{*}{$\begin{array}{c}\text { V/H } \\
\text { Cap* }\end{array}$} & \multicolumn{3}{|c|}{ Overflow } & \multicolumn{3}{|c|}{ Wirelength } & \multirow{2}{*}{$\begin{array}{c}\text { Runtime } \\
\text { (s) }\end{array}$} \\
\hline & & & & BIP*** & $\mathbf{A I P} * *$ & Dec. $\%$ & $\mathbf{W}(\mathbf{m m})$ & $\mathbf{W}^{\prime}(\mathbf{m m})$ & Inc. \% & \\
\hline Cnt100 & 760 & $5 \times 5$ & $15 / 15$ & 18 & 12 & -33.33 & 46376 & 46961 & +1.26 & 2.01 \\
\hline Cnt1000 & 8150 & $20 \times 20$ & $18 / 18$ & 54 & 13 & -75.93 & 1684936 & 1688686 & +0.22 & 19.53 \\
\hline M32_my & 7150 & $20 \times 20$ & $22 / 22$ & 252 & 132 & -47.62 & 962017 & 969448 & +0.77 & 2.72 \\
\hline Gfsm300 & 4154 & $20 \times 20$ & $15 / 15$ & 52 & 26 & -50.0 & 727760 & 725522 & -0.31 & 4.71 \\
\hline Sony_1 & 24847 & $40 \times 40$ & $57 / 57$ & 442 & 419 & -5.2 & 9940513 & 9928071 & -0.13 & 193.09 \\
\hline Toshiba & 16444 & $30 \times 30$ & $61 / 61$ & 387 & 227 & -41.34 & 4264968 & 4259167 & -0.14 & 63.87 \\
\hline
\end{tabular}

Table 4 Experimental results compared with Dragon on IBM-PLACE benchmarks

\begin{tabular}{|c|c|c|c|c|c|c|c|c|c|c|}
\hline \multirow{2}{*}{$\begin{array}{c}\text { Circui } \\
\text { ts }\end{array}$} & \multirow{2}{*}{ \#Cells } & \multirow{2}{*}{ Grids } & \multirow{2}{*}{$\begin{array}{l}\text { V/H } \\
\text { Cap }\end{array}$} & \multicolumn{3}{|c|}{ Overflow } & \multicolumn{3}{|c|}{ Wirelength } & \multirow{2}{*}{$\begin{array}{c}\text { Runtime } \\
\text { (s) }\end{array}$} \\
\hline & & & & BIP & AIP & Dec. $\%$ & $\mathbf{W}(\mathbf{m m})$ & $\mathbf{W}^{\prime}(\mathbf{m m})$ & Inc. \% & \\
\hline Ibm01 & 12,036 & $20 \times 20$ & $27 / 45$ & 156 & 132 & -15.38 & 5171658 & 5118393 & -1.03 & 2.94 \\
\hline Ibm02 & 19,062 & $25 \times 25$ & $54 / 85$ & 597 & 552 & -7.53 & 16403624 & 16422055 & +0.11 & 12.95 \\
\hline Ibm03 & 21,924 & $30 \times 30$ & $36 / 49$ & 413 & 363 & -12.10 & 14193033 & 14210763 & +0.12 & 7.08 \\
\hline Ibm04 & 26,346 & $35 \times 35$ & $38 / 52$ & 337 & 275 & -18.40 & 16057004 & 16093988 & +0.23 & 6.91 \\
\hline Ibm05 & 28,146 & $40 \times 40$ & $67 / 110$ & 546 & 415 & -23.99 & 42195069 & 42230608 & +0.08 & 13.49 \\
\hline
\end{tabular}




\section{References}

[1] A. E. Dunlop and B.W. Kernighan, "A procedure for placement of standard cell VLSI circuits," IEEE Trans. Computer Aided Design, vol. 4, pp. 92-98, Jan. 1985.

[2] H. Eisenmann and F. M. Johannes, "Generic global placement and floorplanning," in Proc. Design Automation Conf., 1998, pp. 269-274.

[3] Saab et.al, "A fast clustering-based Min-cut placement algorithm with simulated-annealing performance," VLSI Design: Int. J. Custom-Chip Design, Simulation, Testing, vol. 5, no. 1, pp. 37-48, 1996.

[4] G. Meixner and U. Lauther, "Congestion-driven placement using a new multi-partitioning heuristic," in Proc. Int. Conf. Computer-Aided Design, Nov. 1990, pp. 332-335.

[5] Zhuoyuan Li, Weimin $\mathrm{Wu}$, Xianlong Hong, Jun Gu, "Incremental placement algorithm for standard-cell layout", Circuits and Systems, 2002 IEEE International Symposium on , Volume: 2 , 2002, Page(s): 883 -886.

[6] Wenting Hou, Hong Yu, Xianlong Hong, Yici Cai, Weimin Wu, Jun Gu, Kao W.H., "A new congestion-driven placement algorithm based on cell inflation", Design Automation Conference, 2001. Proceedings of the ASP-DAC 2001. Asia and South Pacific , 2001, Page(s): 605 -608
[7] Xiaojian Yang; Kastner, R.; Sarrafzadeh, M., "Congestion reduction during placement based on integer programming", Computer Aided Design, 2001. ICCAD 2001. IEEE/ACM International Conference on , 2001, Page(s): 573 -576

[8] Maogang Wang; Xiaojian Yang; Sarrafzadeh, M., "Congestion minimization during placement", Computer-Aided Design of Integrated Circuits and Systems, IEEE Transactions on, Volume: 19 Issue: 10 , Oct. 2000, Page(s): 1140 -1148

[9] Maogang Wang; Sarrafzadeh, M., "Modeling and minimization of routing congestion", Design Automation Conference, 2000. Proceedings of the ASP-DAC 2000. Asia and South Pacific , 2000, Page(s): 185 -190

[10] Maogang Wang; Sarrafzadeh, M., "On the Behavior of Congestion Minimization During Placement", International Symposium on Physical Design, April 1990, Page(s): 145-150

[11] O. Coudert, J. Cong, S. Malik, M. Sarrafzadeh, "Incremental CAD", Computer Aided Design, 2000. ICCAD-2000. IEEE/ACM International Conference on , 2000, Page(s): 236 $-243$

[12] J. Cong and M. Sarrafzadeh, "Incremental Physical Design", Proc. International Symposium on Physical Design, San Diego, California, April 2000, Page(s): 84-92

[13] http://er.cs.ucla.edu/benchmarks/ibm-place/

[14] http://er.cs.ucla.edu/Dragon/ 